\title{
Application of Optimal Control to Tuberculosis Model with Parameter Estimations: Bayesian Approach
}

\author{
Goodluck M. Mlay ${ }^{1 *}$ and Alfred K. Hugo ${ }^{2}$ \\ ${ }^{* 1}$ Department of Mathematics, University of Dar es Salaam, P. O. Box 35062, Dar es Salaam, \\ Tanzania \\ ${ }^{2}$ Department of Mathematics and Statistics, University of Dodoma, P. O. Box 338, Dodoma, \\ Tanzania \\ E-mail addresses: goodluckmlay@gmail.com; alfredkhugo@gmail.com \\ *Corresponding author
}

Received 20 Jun 2020, Revised 5 May 2021, Accepted 6 May 2021, Published May 2021

DOI: https://dx.doi.org/10.4314/tjs.v47i2.25

\begin{abstract}
In this paper, one-strain tuberculosis (TB) model with two control mechanisms, education campaigns and chemoprophylaxis of TB-infected patients, was studied to determine their effects on the reduction of latent and active TB cases. In the case of analysis, boundedness and positivity of the model solutions were carried out to determine the biological feasibility of the study. Besides, the calibration of the parameters by utilizing the identifiability technique through the Markov chain Monte Carlo (MCMC) was thoroughly analysed. The optimum conditions for controlling TB were derived from the Pontryagin Maximum Principle. The numerical simulations were carried out using the forward-back sweep method with the help of the Runge-Kutta fourthorder numerical schemes. Simulation results showed that the education campaigns strategy is more effective in reducing TB infections than the chemoprophylaxis of TB-infected individuals. The combination of the two control strategies reduces a significant number of infections than when each strategy is used on its own. To minimize the transmission of TB from the community, we recommend the education campaigns strategy be a focal point and treatment of latent TB to be paired with the treatment of active TB cases.
\end{abstract}

Keywords: Tuberculosis, Education campaigns, Chemoprophylaxis, MCMC.

\section{Introduction}

Tuberculosis (TB) is a highly contagious airborne disease caused by bacillus Mycobacterium tuberculosis (MTB). Among the infectious diseases caused by a single infectious agent, TB is globally ranked the ninth with an estimate of 1.3 million deaths and 10.4 incident cases recorded in 2016, the tally which is above HIV/AIDS (WHO 2017). The TB burden is high in developing countries due to poor nutrition, the resurgence of HIV and overcrowded accommodation (Mlay et al. 2015, WHO 2017). TB retards the economy of the developing world as it affects more men than women and specifically the productive working group ageing between 15 and 59 years (Agusto 2009, Mlay et al. 2015, WHO 2017). The typical TB affects the lungs (pulmonary TB). However, it can also affect other parts of the body and organs (extrapulmonary TB) such as the central nervous system and bones (WHO 2012).

The major focus of this particular study was on pulmonary TB. Pulmonary TB is a bacterial lung infection that can cause various symptoms, including chest pains, shortness of 
breath and severe coughing. Also, if someone does not receive treatment on time, it can be life-threatening. In this case, an infectious individual expels air that contains the minute droplets of MTB by coughing, sneezing, speaking, or singing (Castillo-Chavez and Song 2004). An individual gets infected with TB when he/she inhales air containing MTB droplets. Once infected, an individual can progress to active TB via two routes: either directly to the infectious state via fast route when the immunity of the body is compromised or slow route through the latent stage of the disease (Ozcaglar et al. 2012). A chance of being infectious is $10 \%$, while $90 \%$ of individuals infected with TB will remain noninfectious for the rest of their lives (Castillo-Chavez and Song 2004, Ozcaglar et al. 2012). The clinical signs of active TB include excessive coughing and coughing up sputum, general tiredness, night sweats, chest pains, fever, weight loss, short breaths and loss of appetite (Cohen and Murray 2004). Treatment of active TB does not confer permanent immunity. Once recovered from $\mathrm{TB}$, an individual may revert to the latent stage. TB dynamics are complex in such a way that even reducing the basic and the effective reproduction numbers below one do not guarantee the clearance of the disease from the community. Modelling of the TB epidemics has become the means to study the complex dynamics of the disease to advise the public health policymakers to construct the appropriate controls and intervention strategies to fight against TB infections (Hattaf et al. 2009, Mlay et al. 2015).

Hattaf et al. (2009), formulated an optimal model of TB with exogenous reinfection by introducing a control mechanism that prevents exogenous reinfection as the result of preventing contacts between infectious and infected individuals in the latent group. The results showed that the number of infectious individuals in the one-year frame is significantly reduced when the control is imposed on latently infected individuals than when control is not used. An optimal control model of TB with treatment, a case study of Angola was formulated by Silva and Torres (2012) to include the mechanisms of helping infectious individuals to complete the treatment and reducing the number of individuals with persistent latent TB. The successful implementation of these controls prevents the enviable number of new and reinfected TB cases. Athithan and Ghosh (2015) formulated an optimal model of TB with case treatment and detection. They treated the case detection parameter as a variable (time-dependent). As time evolves, they managed to show that the case detection reduces the significant number of infected individuals when treated as a variable than when is taken as a constant parameter. Jung et al. (2002) came up with two strain tuberculosis model in which the treatment efforts (case holding and case finding) were introduced. They found that the program that follows these treatment efforts reduces a remarkable number of latent and infectious individuals with resistant TB cases.

In this article, we modified a TB model of Mlay et al. (2014) by assuming that some infected individuals are immune-compromised as a result of progressing faster to active TB. Two control mechanisms, education campaigns and chemoprophylaxis (treatment of latent TB) are incorporated into the model as efforts to reduce the number of individuals who are latently and actively infected with TB. Besides, we estimated model parameters using Markov Chain Monte Carlo (MCMC) technique to determine the efficiency and accuracy of model parameters. The identifiability technique was used in the calibration of parameters as the study misses the actual data. Solonen et al (2013) has applied the same technique.

The MCMC technique is widely used in various areas of research. Gilks et al. (1995) used it in stochastic non-linear dynamic systems in various fields of physics, biology, chemistry, and computational finance; while Mbalawata et al. (2014) applied MCMC in stochastic models and Solonen et al. (2013) 
applied it in SEIR epidemic model. The MCMC algorithm usually works by generating a sequence of random draws from the target distribution which converge to prior distribution (Gilks et al. 1995, Haario et al. 2001, Gelman et al. 2013). The generated samples of the data $(y)$ are therefore used to determine the properties of the distribution of each parameter. The prior information is usually termed as a prior distribution $p(\phi)$, where $\phi$ stands for model parameters that are randomly considered. In the Bayesian inference approach, the parameter is estimated by employing posterior distribution $\pi(\phi \mid y)$ as it is pointed in Mbalawata et al. (2014). The posterior distribution $\pi(\phi \mid y)$ is defined as

$$
\pi(\phi \mid y)=\frac{l(y \mid \phi) p(\phi)}{\int l(y \mid \phi) p(\phi) d \phi},
$$

whereas $l(y \mid \phi)$ represents the likelihood function that contains the necessary information given by the data, $p(\phi)$ is the prior distribution and denominator, $\int l(y \mid \phi) p(\phi) d \phi$ is known as normalization constant that ensures posterior distribution integrates to one.

The most well-known algorithms in MCMC methods are the Metropolis, MetropolisHastings and Gibbs sampler algorithms (Gilks et al. 1995). The Metropolis algorithm usually generates a sequence of random draws from the approximate distribution which converge to $\pi(\phi \mid y)$ (Gilks et al. 1995, Haario et al. 2001, Gelman et al. 2013). The MetropolisHastings algorithm is a generalization of the random walk Metropolis algorithm that uses an accept/reject rule to converge to the target distribution $\pi(\phi)$ (Gilks et al. 1995). The new candidate $\phi^{*}$ is created from proposal distribution $q(. \mid \phi)$ that contains the information from the previous values $\phi$. The MCMC algorithm produces a chain of values that depend on the previous iterations (Chib and Greenberg 1995). The Metropolis-
Hastings (MH) algorithm works by sampling a candidate point $\phi^{*}$ from a proposal distribution $q(. \mid \phi)$ and then accepting the point with an acceptable probability of $\alpha=\min \left(1, \frac{\pi\left(\phi^{*}\right) q\left(\phi_{\text {old }} \mid \phi^{*}\right)}{\pi\left(\phi_{\text {old }}\right) q\left(\phi^{*} \mid \phi_{\text {old }}\right)}\right) \quad$ (Gilks et al. 1995).

In general, the proposal distributions used in MCMC algorithms should result in well mixing of chains and converge at a suitable acceptance rate. The determination of the most suitable proposal distribution for a particular target distribution is a very important aspect. Moreover, it is a difficult task because it involves many trials and errors (Mbalawata et al. 2014). Markov chain is said to converge when the unique distribution has been achieved and assessed by how well the chain is mixed. The good mixing of the chain means the degree to which the Markov chain explores the support of the posterior distribution. The convergence can be assessed through statistical and graphical tools such as Trace or time series, two-dimensional parameters and or auto-correlation plots.

\section{Materials and Methods}

In this section, a one-strain deterministic compartmental model was constructed by modifying the model of Mlay et al. (2014). The modification task was done by fusing the infectious classes $I_{1}$ and $I_{2}$ to a single infectious class $I$ that leads to Susceptible-VaccinatedExposed-Infectious-Treated (SVEIT) model. Besides, we assumed that some susceptible individuals progress faster to infectious class and an individual can die due to disease while is under treatment. Susceptible individuals are recruited by birth at a rate of $\lambda$ and come into contact with infectious individuals, $I$ at percapita infection rate $\beta$. A fraction $\varepsilon$ of susceptible individuals who are immunocompromised join directly to the infectious group I. The proportion, $\rho$ of babies, are vaccinated at birth while the remaining 
proportion $(1-\rho)$ is left out to join the susceptible class. Vaccinated babies lose immunity and become susceptible at a percapita rate $\theta$, from which $\frac{1}{\theta}$ is the mean expiry period of a vaccine. Latently infected individuals are endogenously reactivated and progress to active TB at the per-capita rate $\varepsilon$. The infectious individuals progress to the treatment group at the per-capita rate $v$. TB does not confer permanent immunity. Once treated, an individual may receive another episode of disease and relapse back to the latent group at a reduced infection rate of $\gamma$. Infectious and treated individuals are assumed to die with disease-induced mortality rates of $\delta_{1}$ and $\delta_{2}$, respectively, while the rest classes die naturally at a per-capita rate $\mu$. All variables and parameters are assumed to be non-negative.

Besides, we formulate the model by making the following assumptions:

a) All individuals are susceptible to $\mathrm{TB}$, mixing homogeneously and have an equal chance to be infected with $\mathrm{TB}$ regardless of age, sex and social status.

b) TB vaccine is administered only once to susceptible individuals and usually provided to the newborn.

c) Natural recovery is negligible and hence ignored.

d) Recovery of an active TB individual is only through treatment.

\section{Model equations}

The model description and assumptions made in the section of materials and methods lead to the following system of ordinary differential equations:

$$
\begin{aligned}
& \frac{d S}{d t}=(1-\rho) \lambda N-\frac{\beta S I}{N}-\mu S+\theta V, \\
& \frac{d V}{d t}=\rho \lambda N-(\mu+\theta) V,
\end{aligned}
$$

$\frac{d L}{d t}=\frac{\beta S I}{N}+\gamma \frac{\beta T I}{N}-(\mu+\varepsilon) L$,

$\frac{d I}{d t}=\varepsilon L-\left(v+\mu+\delta_{1}\right) I$,

$\frac{d T}{d t}=v I-\gamma \frac{\beta T I}{N}-\left(\mu+\delta_{2}\right) T$,

$N=S+V+L+T+I$.

\section{Positivity and boundedness of solutions}

The solutions of the model (1) denoted by $\square_{+}^{5}$ are such that, the set of the points $x_{i}=\left(x_{1}, x_{2}, x_{3}, x_{4}, x_{5}\right)$ in $\square_{+}^{5}$ has positive coordinates. We consider the system of Equations (1) with the initial values $x^{0}=\left(x_{1}^{0}, x_{2}^{0}, x_{3}^{0}, x_{4}^{0}, x_{5}^{0}\right)$. Using the approach of Lungu et al. (2013), we formulate the following lemma:

Lemma 1. The system (1) can be transformed into the following differential inequalities

$$
\begin{gathered}
\frac{d x_{i}}{d t} \geq A_{i} x_{i}+\sum_{j=1}^{n} B_{i j} x_{j}+\varepsilon, \\
(i=1,2,3, \ldots, n),
\end{gathered}
$$

where $B_{i j} \geq 0, \varepsilon \geq 0$. If $x_{i}(0) \geq \varepsilon$ then $x_{i}(t) \geq 0$ for all $t>0$ and $1 \leq i \leq n$. We assume that $\varepsilon>0$. For the case, $\varepsilon=0$ it approximates the system with the sequence $\varepsilon=\varepsilon_{k} \downarrow 0$.

Proof. By contradiction

Suppose $x_{i}(0) \geq \varepsilon$ for $1 \leq i \leq 5$ is not true. Hence there exists a smallest number $t_{0}>0$ such that $x_{i}(t)>0$ for $1 \leq i \leq n, 0 \leq t \leq t_{0}$, $x_{i}\left(t_{0}\right)=0$ for at least one $i$ say $i=i_{0}$. Then $x_{i 0}$ is a decreasing function at $t=t_{0}$, so that

$$
\frac{d x_{i 0}}{d t}\left(t_{0}\right) \leq 0 \text {. }
$$


At $x_{i 0}(t)$, the differential inequality becomes

$$
\frac{d x_{i 0}}{d t}\left(t_{0}\right) \geq \sum_{j=1}^{n} B_{i j} x_{j}+\varepsilon \geq \varepsilon>0 .
$$

This contradicts our Lemma (1). For the state variables in our model, we always take

$S(0) \geq 0, V(0) \geq 0, L(0) \geq 0, I(0) \geq 0, T(0) \geq 0$. (5

Thus in the region, $\square_{+}^{5}$ the model is epidemiologically and mathematically wellposed $\square$

$$
\begin{aligned}
& \frac{d S}{d t}=(1-\rho) \lambda N-\left(1-u_{1}\right) \frac{\beta S I}{N}-\mu S+\theta V, \\
& \frac{d V}{d t}=\left(1+u_{1}\right) \rho \lambda N-(\mu+\theta) V, \\
& \frac{d L}{d t}=\left(1-u_{1}\right) \frac{\beta S I}{N}+\left(1-u_{1}\right) \gamma \frac{\beta T I}{N}-\left(1-u_{2}\right) \varepsilon L-\mu L, \\
& \frac{d I}{d t}=\left(1-u_{2}\right) \varepsilon L-\left(1+u_{1}\right) v I-\left(\mu+\delta_{1}\right) I, \\
& \frac{d T}{d t}=\left(1+u_{1}\right) v I-\left(1-u_{1}\right) \gamma \frac{\beta T I}{N}-\left(\mu+\delta_{2}\right) T .
\end{aligned}
$$

The incorporated controls are used in making decisions involving complex biological situations (Lenhart and Workman 2007). It determines the optimal level of the intervention strategy that is preferred in reducing the spreads of $\mathrm{TB}$. The objective functional is minimized subject to the system of ordinary differential equations in (6) and is expressed as

$$
J=\min _{u_{1}, u_{2}} \int_{0}^{t_{f}}\left(A_{1} L+A_{2} I+\frac{1}{2} B_{1} u_{1}^{2}+\frac{1}{2} B_{2} u_{2}^{2}\right) d t,
$$

where $t_{f}$ is the final time, $A_{1} L$ and $A_{2} I$ are the social relative costs associated with controlling TB to the individuals in latent and infectious classes, respectively, while $B_{1}$ and $B_{2}$ are relative weights associated with respective control measures $u_{1}$ and $u_{2}$. The objective functional (7) is involved in minimizing the costs of controls. In this paper, a quadratic function that satisfies the optimality conditions is considered. Thus, there exist optimal controls $u_{1}^{*}(t)$ and $u_{2}^{*}(t)$ such that

$J\left(u_{1}^{*}(t), u_{2}^{*}(t)\right)=\min \left\{J\left(u_{1}(t), u_{2}(t) \mid u_{1}(t), u_{2}(t) \in U\right)\right\}$,

where $U=\left\{\left(u_{1}(t), u_{2}(t)\right)\right\}$ is Lebesgue measurable, $a_{i} \leq\left(u_{1}, u_{2}(t)\right) \leq b_{i}, i=1, \cdots 5, a_{i}=0$, $b_{i}=1, t \in\left[0, t_{f}\right]$ is the closed set and are evaluated under Lebesgue measurable control. The necessary conditions that satisfy the optimal control are developed by Pontryagin's Maximum Principle (Lenhart and Workman 2007). The principle works by converting the system of Equations (6) and (7) into a pointwise minimization problem known as a Hamiltonian, $H$ function such that 


$$
\begin{aligned}
H= & A_{1} L+A_{2} I+\frac{1}{2} B_{1} u_{1}^{2}+\frac{1}{2} B_{2} u_{2}^{2}+ \\
& \lambda_{1}\left\{(1-\rho) \lambda N-\left(1-u_{1}\right) \frac{\beta S I}{N}-\mu S+\theta V\right\}+ \\
& \lambda_{2}\left\{\left(1+u_{1}\right) \rho \lambda N-(\mu+\theta) V\right\}+ \\
& \lambda_{3}\left\{\left(1-u_{1}\right) \frac{\beta S I}{N}+\left(1-u_{1}\right) \gamma \frac{\beta T I}{N}-\left(1-u_{2}\right) \varepsilon L-\mu L\right\}+ \\
& \lambda_{4}\left\{\left(1-u_{2}\right) \varepsilon L-\left(1+u_{1}\right) v I-\left(\mu+\delta_{1}\right) I\right\}+ \\
& \lambda_{5}\left\{\left(1+u_{1}\right) v I-\left(1-u_{1}\right) \gamma \frac{\beta T I}{N}-\left(\mu+\delta_{2}\right) T\right\},
\end{aligned}
$$

where $\lambda_{i}, i=1,2, \cdots, 5$ are the co-state variables associated with state variables $S, I, V, L$ and $T$. The adjoint equations are obtained by using relation

$$
\frac{d \lambda_{i}}{d t}=-\frac{\partial H}{\partial x_{i}}
$$

with transversality condition

$$
\lambda_{i}\left(t_{f}\right)=0 \text {. }
$$

From (8) we obtain the following adjoint equations:

$$
\begin{aligned}
& \frac{d \lambda_{1}}{d t}=\lambda_{1}\left(\mu+\frac{\beta I}{N}\left(1-u_{1}\right)\right)-\frac{\lambda_{3}}{N}\left(1-u_{1}\right) \beta I, \\
& \frac{d \lambda_{2}}{d t}=-\lambda_{1} \theta+\lambda_{2}(\mu+\theta), \\
& \frac{d \lambda_{3}}{d t}=\left(1-u_{2}\right) \varepsilon\left(\lambda_{3}-\lambda_{4}\right)+\lambda_{3} \mu-A_{1}, \\
& \frac{d \lambda_{4}}{d t}=-A_{2}+\frac{\beta S}{N}\left(1-u_{1}\right)\left(\lambda_{1}-\lambda_{3}\right)+\frac{\beta T}{N}\left(1-u_{1}\right)\left(\lambda_{5}-\lambda_{3}\right)+\left(1+u_{1}\right) v\left(\lambda_{4}-\lambda_{5}\right)+\lambda_{4}\left(\mu+\delta_{1}\right) \\
& \frac{d \lambda_{5}}{d t}=\lambda_{5}\left(\mu+\delta_{2}\right)+\left(\lambda_{5}-\lambda_{3}\right) \frac{\left(1-u_{1}\right) \gamma \beta I}{N} .
\end{aligned}
$$

The optimality of the control model (6) is obtained by using

$$
u_{i}^{*}(t)=\frac{\partial H}{\partial u_{i}}=0
$$

where $i=1,2$. The solutions of $u_{1}^{*}(t)$ and $u_{2}^{*}(t)$ are presented in a compact form as

$$
u_{1}^{*}(t)=\max \left\{0, \min \left\{1, \frac{\left(\lambda_{4}-\lambda_{5}\right) v I N+\left(\lambda_{3}-\lambda_{1}\right) \beta S I+\left(\lambda_{3}-\lambda_{5}\right) \gamma \beta I T-\lambda_{2} \rho N^{2}}{N B_{1}}\right\}\right\},
$$


Mlay and Hugo - Application of optimal control to tuberculosis model with parameter ...

$$
u_{2}^{*}(t)=\max \left\{0, \min \left\{1, \frac{\varepsilon L\left(\lambda_{4}-\lambda_{3}\right)}{B_{2}}\right\}\right\} .
$$

\section{Results and Discussion}

The parameter identifiability for model (1) has been manipulated by the means of Gaussian noise through the Delayed Rejection Adaptive Metropolis (DRAM) algorithm. The MCMC samples are analyzed via scattering and auto-correlation plots. The graphical method shows the correctness of the distribution of the chain and henceforth, we determine the goodness of the performance of the MCMC method. The estimated parameter values are therefore presented in Table 1 and are used for numerical simulations of the model. The Geweke test is used in the comparison of the mean of the first $10 \%$ of the chain samples to the next second half of the chain (Geweke and Tanizaki 2003), and its outcomes are therefore presented in Table 1.

Table 1: MCMC estimated parameter values of the model

\begin{tabular}{ccccccc}
\hline Symbol & Value & Estimated & Mean & Std & MCMC error & Geweke \\
\hline$\lambda$ & 0.05 & 0.049877 & 0.050168072 & 0.000858465 & 0.00011428 & 0.989731486 \\
$\beta$ & 2.58 & 2.580579 & 2.578914906 & 0.013068771 & 0.001884119 & 0.999354325 \\
$\rho$ & 0.4 & 0.402655 & 0.400770992 & 0.008978622 & 0.001327865 & 0.999822737 \\
$\theta$ & 0.1 & 0.1009 & 0.100746486 & 0.002716445 & 0.000362394 & 0.988338865 \\
$\varepsilon$ & 0.03 & 0.029985 & 0.030113728 & 0.000387371 & 0.00004 & 0.9955884 \\
$\mu$ & 0.02 & 0.019083 & 0.019437045 & 0.001083897 & 0.000146612 & 0.963203722 \\
$\delta_{1}$ & 0.3 & 0.298597866 & 0.294376438 & 0.012281125 & 0.001617703 & 0.960811576 \\
$\nu$ & 0.3 & 0.300757638 & 0.304299461 & 0.01721474 & 0.002465443 & 0.916977296 \\
$\gamma$ & 0.2 & 0.192241199 & 0.195692833 & 0.078573245 & 0.012212649 & 0.587932159 \\
$\alpha$ & 0.1 & 0.099035081 & 0.09979694 & 0.00821952 & 0.001151098 & 0.912942282 \\
$\delta_{2}$ & 0.2 & 0.201833459 & 0.203942958 & 0.007165459 & 0.001025618 & 0.949477431 \\
\hline
\end{tabular}

The convergences of each parameter generated by MCMC are presented in Figures 1, 2, 3 and 4. The correctness and goodness of the performance of the MCMC results are analyzed by using scatter, autocorrelation, trace, and marginal distribution plots. Figure 1 shows the samples for the parameters in 10,000 iterations and parameters such as $\lambda, \beta$, $\rho, \theta, \varepsilon, \mu$ and $\alpha$ show a good mixing than the rest in the list of parameters displayed in Table 1. Pair plots of parameters reveal good relationships as many parameters indicate positive and strong correlations as shown in Figure 4. The convergences of MCMC sample parameters are therefore graphically visualized by plotting autocorrelation plots as shown in Figure 2. The convergence oscillates up and down and finally stabilizes at around zero with 60 lags as shown in Figure 2. The sample marginal distribution plots with mean and original parameters are depicted in Figure 3 and provide the promising values of the parameters which show the estimated values tend to be close to the original values. 

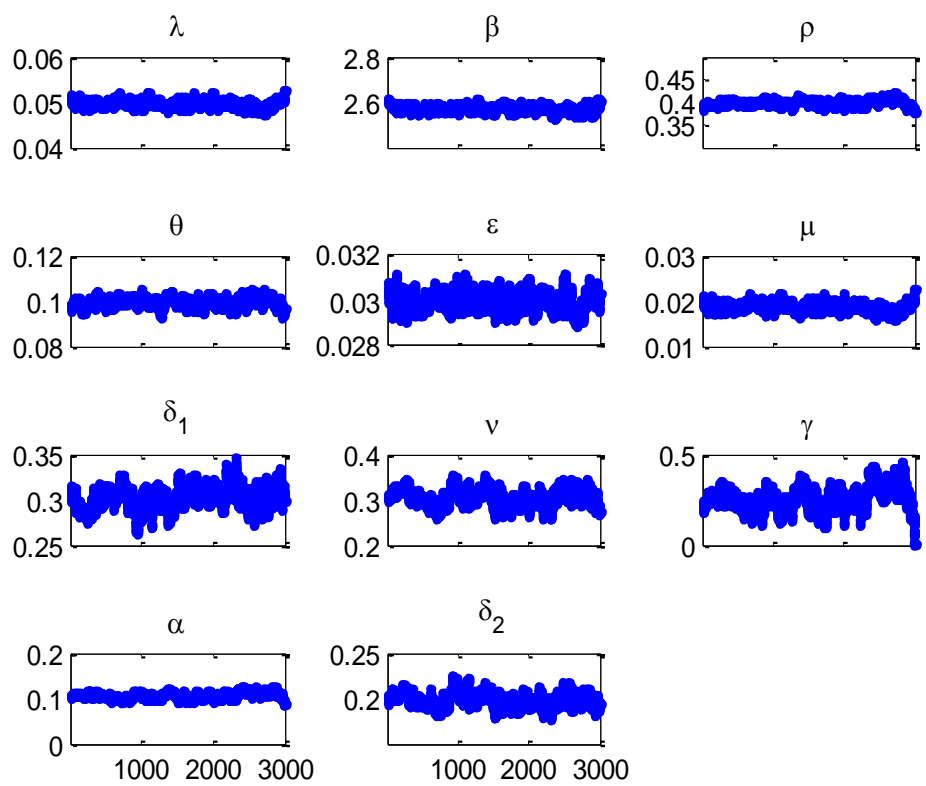

Figure 1: Markov Chain Monte Carlo (MCMC) scatter plots. The vertical axis represents samples and the horizontal axis represents the number of iterations.
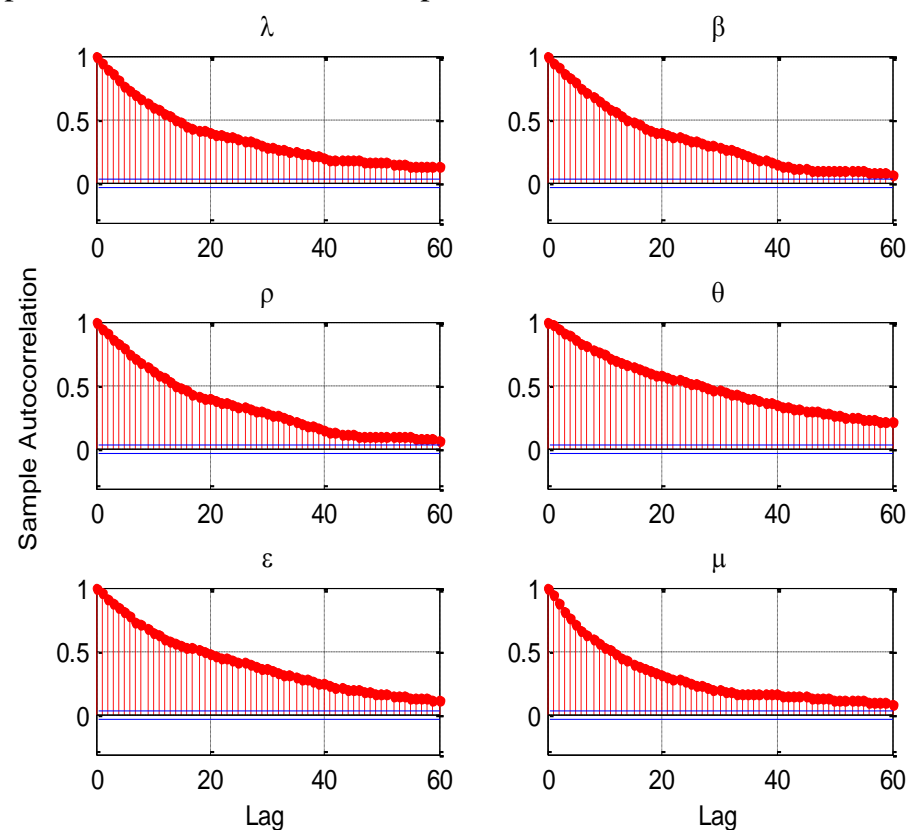

Figure 2: $\mathrm{MCMC}$ autocorrelation plots for some of the parameters. The vertical axis represents samples, while the horizontal axis represents the number of iterations. 

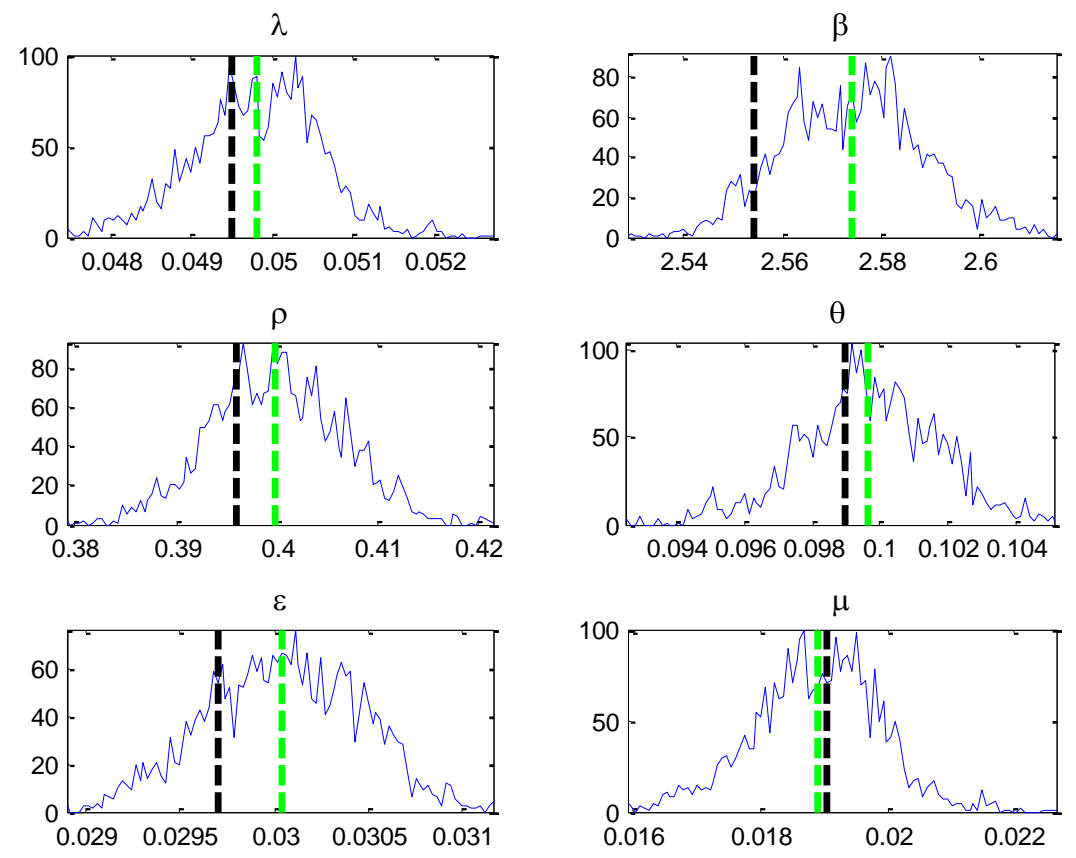

Figure 3: Marginal distribution plots and a mean of MCMC samples. The green colour represents the sample mean, while the black colour represents the original parameter values. The $\mathrm{x}$-axis represents the parameter distribution, while the $\mathrm{y}$-axis represents the number of iterations.
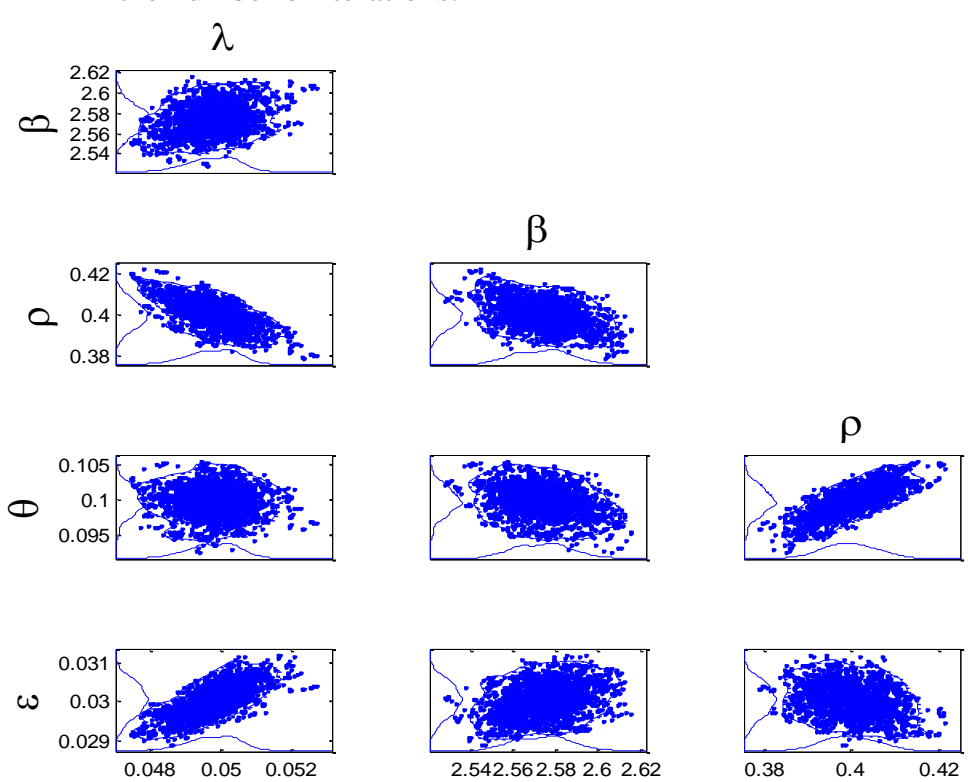

$\theta$

Figure 4: A plot showing pair correlation of parameters. 
The estimated parameters were therefore used to solve and simulate state optimal control model (6) together with adjoint Equations (11), ..., (15) through the RungeKutta forward-backward sweep techniques. The adjoint Equations (11), (12), (13), (14) and (15) were solved by the fourth-order RungeKutta scheme using the forward solution of the state equations in (6). While optimality conditions are satisfied through the convex update of the previous control values. The numerical results are now presented in the following Figures 5 to 7.

In particular, the control profile of Figure 5 shows that the control, $u_{1}$ remains at 1 for 0.55
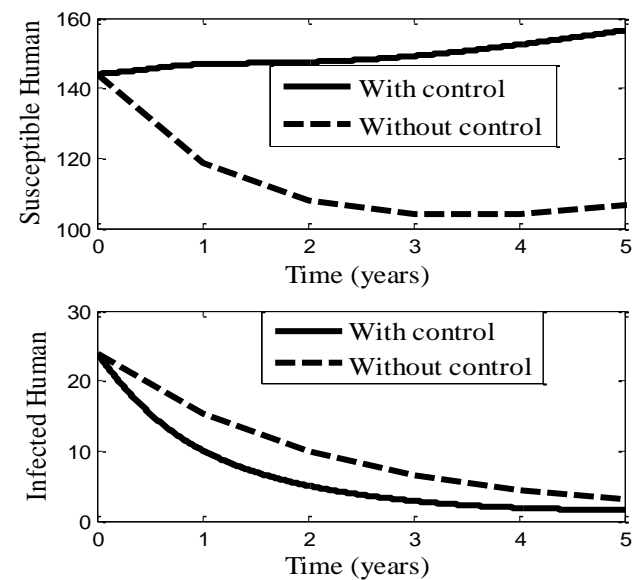

Figure 5: Effect of the education campaigns, $u_{1}$ and chemoprophylaxis of latently infected $u_{2}$.
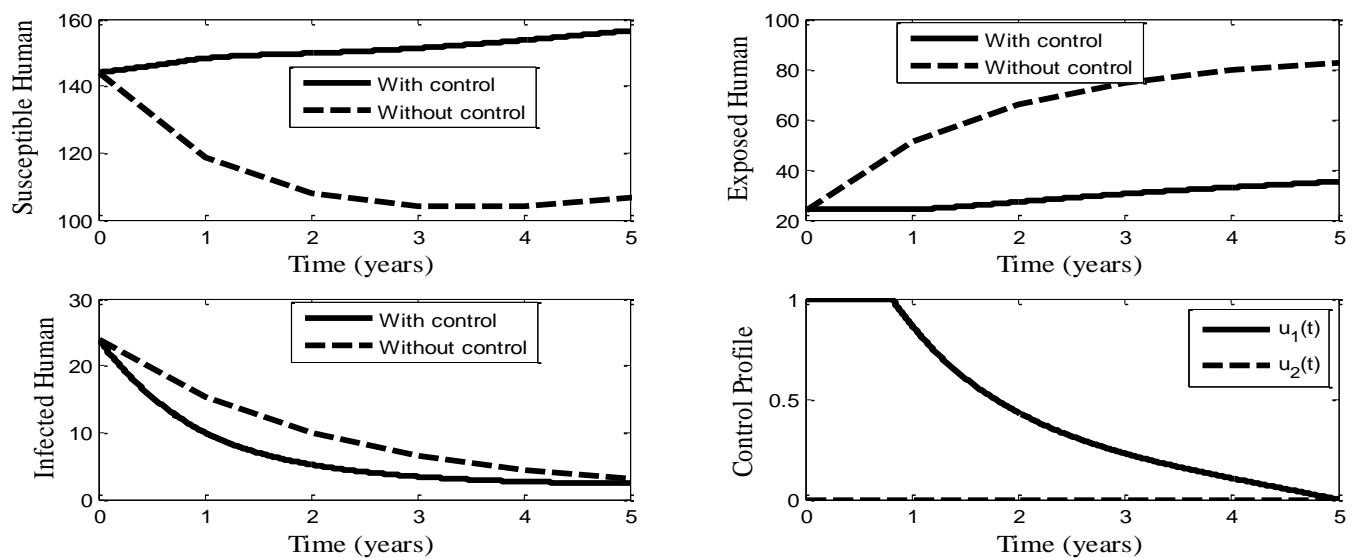

Figure 6: Effect of the education campaigns, $u_{1}$. 
Mlay and Hugo - Application of optimal control to tuberculosis model with parameter ...
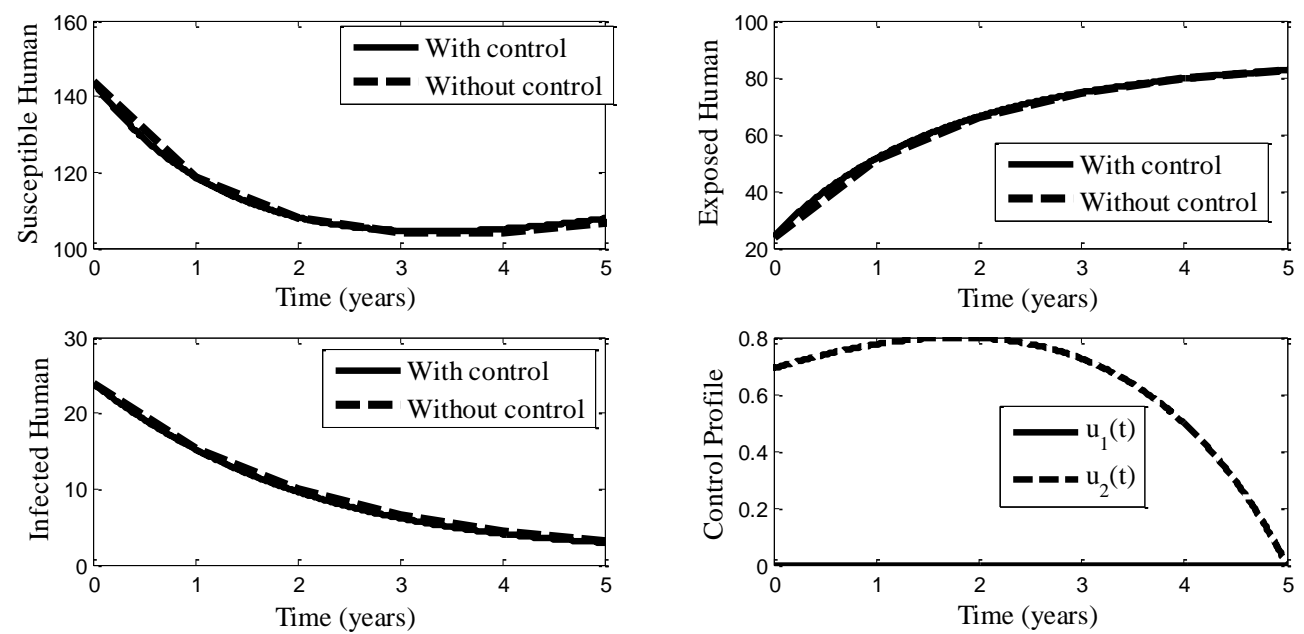

Figure 7: Effect of chemoprophylaxis of latently infected, $u_{2}$.

\section{Conclusion}

The dynamical model for the transmission of TB has been developed and analyzed. In this paper, the main focus was to estimate parameters by using the Bayesian approach via Markov Chain Monte Carlo (MCMC) as well as solving the time-dependent control problem to seek optimal control measures to combat TB infections and transmissions. The output of MCMC shows good correlation as well as generated parameter values used in numerical simulations. The optimal control numerical simulations which are based on ForwardBackward Sweep Method (FBSM) were carried out and the results were presented in Figure 5 to Figure 7. Two control strategies were involved; these include education campaigns, $u_{1}$ and chemoprophylaxis of latently infected individuals, $u_{2}$. Numerical simulations showed that the combination of education campaigns and chemoprophylaxis was the best strategy in controlling TB than when each strategy was taken alone. From the findings of this study, it is concluded that the mathematical model based on parameter estimation and the optimal control theory lead to important results due to accurate and precise parameter values used for numerical simulations which in turn reflect economic consequences of the studied disease.
Conflict of Interest: No conflict of interest.

\section{References}

Agusto F 2009 Optimal chemoprophylaxis and treatment control strategies of a tuberculosis transmission model. World J. Model. Simul. 5(3): 163-173.

Athithan S and Ghosh M 2015 Optimal control of tuberculosis with case detection and treatment. World J. Model. Simul. 11: 111122.

Castillo-Chavez C and Song B 2004 Dynamical models of tuberculosis and their applications. Math. Biosci. Eng. 1(2): 361404.

Chib S and Greenberg E 1995 Understanding the metropolis-hastings algorithm. The American Statistician 49(4): 327-335.

Cohen T and Murray M 2004 Modeling epidemics of multidrug-resistant $M$. tuberculosis of heterogeneous fitness. Nature Med. 10(10): 1117-1121.

Gelman A, Carlin JB, Stern HS, Dunson DB, Vehtari A and Rubin DB 2013 Bayesian data analysis. Chapman and Hall/CRC Press.

Geweke J and Tanizaki H 2003 Note on the sampling distribution for the metropolis- 
hastings algorithm. Commun. StatisticsTheor. Meth. 32(4): 775-789.

Gilks WR, Richardson S and Spiegelhalter D 1995 Markov chain Monte Carlo in practice. Chapman and Hall/CRC.

Haario H, Saksman E and Tamminen J 2001 An adaptive metropolis algorithm. Bernoulli 7(2): 223-242.

Hattaf K, Rachik M, Saadi S, Tabit Y and Yousfi N 2009 Optimal control of tuberculosis with exogenous reinfection. Appl. Math. Sci. 3(5): 231-240.

Jung E, Lenhart S and Feng Z 2002 Optimal control of treatments in a two-strain tuberculosis model. Discrete and Continuous Dynamical Systems Series B 2(4): 473-482.

Lenhart S and Workman JT 2007 Optimal control applied to biological models. Chapman and Hall/CRC.

Lungu E, Massaro TJ, Ndelwa E, Ainea N, Chibaya S and Malunguza NJ 2013 Mathematical modelling of the HIV/Kaposi's sarcoma coinfection dynamics in areas of high HIV prevalence. Comput. Math. Meth. Med. 2013.

Mbalawata IS 2014 Adaptive Markov chain Monte Carlo and Bayesian filtering for state-space models. $\mathrm{PhD}$ Thesis, Lappeenranta University of Technology.

Mlay GM, Luboobi L, Kuznetsov D and Shahada F 2015 Optimal treatment and vaccination control strategies for the dynamics of pulmonary tuberculosis. Int. J. Adv. Appl. Math. Mechan. 2(3): 196-207.

Mlay GM, Luboobi LS, Kuznetsov D and Shahada F 2014 Dynamics of a onestrain pulmonary tuberculosis model with vaccination and treatment Commun. Math. Biol. Neurosci. 2014.

Ozcaglar C, Shabbeer A, Vandenberg SL, Yener B and Bennett KP 2012 Epidemiological models of Mycobacterium tuberculosis complex infections. Math. Biosci. 236(2): 77-96.

Silva CJ and Torres DF 2012 Optimal control strategies for tuberculosis treatment: a case study in Angola. Numerical Algebra, Control and Optimization 2(3): 601-617.

Solonen A, Haario H, Tchuenche JM and Rwezaura H 2013 Studying the identifiability of epidemiological models using MCMC. Int. J. Biomath. 6(02):1350008.

WHO 2012, 2010/2011 Tuberculosis Global Facts: Global Tuberculosis Report. http://apps.who.int/iris/bitstream/10665/759 38/1/9789241564502 eng.pdf

WHO 2017 Global Tuberculosis Report. https://www.who.int/tb/publications/global _report/gtbr2017_main_text.pdf 